\title{
The Effect of Compression on Natural Graphite Anode Performance and Matrix Conductivity
}

\author{
K. A. Striebel, A. Sierra, J. Shim \\ Environmental Energy Technologies Division \\ Lawrence Berkeley National Laboratory \\ Berkeley, CA 94720 \\ and \\ C.-W. Wang, A. M. Sastry \\ Department of Mechanical Engineering \\ University of Michigan \\ Ann Arbor, MI 48109-2129
}

\begin{abstract}
Anodes for lithium-ion cells were constructed from three types of natural graphite, two coated spherical and one flaky. Anode samples were compressed from 0 to $300 \mathrm{~kg} / \mathrm{cm}^{2}$ and studied in half-cells to study the relations between anode density, SEI formation and anode cyclability. The $\mathrm{C} / 25$ formation of the SEI layer was found to depend on the nature of the graphite and the anode density. Compression of the uncoated graphite lead to an increased conductivity, but only slight improvements in the efficiency of the formation process. Compression of the anodes made from the amorphous-carbon-coated graphites greatly improved both the reversible capacity and first-cycle efficiency. In addition, the fraction of the irreversible charge associated with the surface of the graphite increased with compression, from both an increase in the electrolyte contact as well as compression of the amorphous layer. The cyclability of all of the anodes tended to improve with compression. This suggests that it is the improvement in the conductivity of the anode plays more of a role in the improvement in the cyclability than the formation process.
\end{abstract}




\section{Introduction}

Various forms of graphite are most common as the anode active material in today's Li-ion batteries. Natural graphites (NG) will be favored over the synthetics for use in batteries for transportation applications because of cost, $5-15 \$ / 1 \mathrm{~b}$, vs. 50\$/lb, respectively. In a high-energy battery for an all-electric vehicle (EV), the range of the vehicle will depend directly on the capacity of the cells, or the amount of cycleable lithium inventory in the cell. Any consumption of this lithium at the anode though formation and/or instability of the SEI layer will directly lower cell capacity. In hybrid electric vehicles (HEV), the absolute capacity of the cell is less critical, however, instabilities in the SEI layer that lead an increased thickness in this layer will result in a loss of cell power capability.

Many factors have been shown to influence the behavior of the SEI on a natural graphite anode. The nature of the carbon and the composition of the electrolyte are the most important and the most studied [1,2]. The electrolyte composition will affect the amount of solvent cointercalation [3] that will precede or accompany the formation of the passivating layer on the graphite and the first intercalation of the Li ions into the graphite. EC-based solvents are thought to react to form an SEI layer that prevents solvent intercalation [4]. The nature of the Li salt has been shown to affect the composition of the SEI since it clearly takes part in the formation reaction [5]. Several studies have been carried out on the effect of particle size, morphology and, surface area on the charge consumed by the formation of the SEI. Zaghib et. al report that the amount of SEI formation increases with graphite surface area [6]. Peled [7] and Zaghib [8] examined the SEI dependence on carbon morphology, namely the edge versus basal plane sites. The edge-site SEI was shown to be significantly thicker than the SEI on the basal plane [7]. They also found a composition difference, i.e. a large amount of inorganic LiF-type species in the edge-site SEI with a more organic SEI on the basal planes. Zaghib et al did not study the sites individually, but found a larger irreversible capacity loss (ICL) on thin flaky graphite than thick, despite the lower fraction of edge sites to basal plane sites in the former. The conflicting reports can be explained by the dependence of the SEI formation on the rate of Li transport into the graphite, or the residence time of the $\mathrm{Li}$ ions at the surface of the graphite particle with accessibility to solvent molecules.

While the properties of the SEI created during the anode formation are important, the stability of the SEI during cycling is also critical. Commercial anodes usually contain upwards of $94 \%$ active material, a few percentage of a soft carbon conductive agent, and they are usually 
pressed to about $30 \%$ porosity. It is accepted that these treatments are essential to obtaining highenergy, high-rate and good cycling stability. However, there are conflicting ideas in the literature as to why this is the case. The addition of conductive agents will tend to increase the conductivity of the solid phase in the anode, as well as acting as a wetting agent to keep the electrolyte in the structure. However, conductive additives, such as carbon black, have been implicated as a significant drain on the Li inventory during formation [9]. Compression of the anode to low porosity will also act to improve the conductivity of the solid matrix phase. Takamura et al. suggest that excellent conductivity within the anode structure is critical to the formation of a uniform SEI layer over each graphite particle [10]. This was attributed to a more uniform current distribution over the graphite particles which leads to a more uniform thickness and composition to the SEI. A contrasting report comes from the people at Bellcore who suggest that an anode SEI formed at high rate, instead of a slow steady rate, resulted in anodes that were more stable to the effects of $\mathrm{Mn}$ diffusing form the a $\mathrm{Li}_{1+\mathrm{y}} \mathrm{Mn}_{2-\mathrm{y}} \mathrm{O}_{4}$ cathode at moderate temperatures [11]. The influence of anode conductivity is not clear however, since an analysis of the current distribution over an individual graphite particle will conclude that the conductivity of the graphite is high enough to lead to as much uniformity as you could ask for [12] and that in effect the SEI and the rate of Li insertion into graphites is controlled solely by surface kinetics [13].

Anode compression also decreases the anode porosity, and the ratio of graphite surface area to electrolyte volume. There have been some studies of the effect of anode compression on capacity and efficiency with various graphites. Gnanaraj et al. [14] and Novak et. al. [15] showed a decrease in both capacity and first cycle efficiency with high levels of compression. These were explained by transport limitations within the liquid path as well as particle break-up for the largest pressures. However, complication of unfilled pores due to wettability problems may have played a role in these studies. In previous work from this lab, we studied low-loading anodes, prepared with a natural graphite from Superior Graphite compressed at a range of moderate pressures, and found a decrease of both ICL and reversible capacity with increases in anode density [16]. In addition, cyclability was seen to improve slightly with anode density. The decreased reversible capacity was attributed to increased stress within the graphite sheets, which also slowed down in the Li ion diffusion process [17]. In another work, high-energy cells with pressed electrodes showed a dramatic improvement in cycle-life stability over those with unpressed electrodes [16]. 
The connection between the solid phase conductivity of an electrode and its capacity, efficiency and stability is not well-understood. This is due in part to the empirical nature of conductivity measurements on porous electrodes. Recently a better understanding of the resistivity of anodes with different fractions of various anode particles and the amorphous carbon fraction has been attained though conductivity modeling [18]. In this work, we carried out a systematic investigation between the irreversible capacity loss (ICL), stability (cyclability) and the resistivity of natural graphite anodes prepared from three types of NG and compressed to a range of densities. The three natural graphites were a purified NG from Superior Graphite (SL20) and two NG's from Mitsui Mining with either 6 or 14\% coating of amorphous carbon. The amorphous carbon coating is added to the surface of natural graphite to improve it's resistance to PC solvent intercalation [19-20]. However, in this work the coated graphites lend an added avenue for the investigation of the connections between anode conductivity and performance and the role of an amorphous carbon fraction. The conductivity analysis of these electrodes has already been reported [21]. This paper reports the electrochemical performance: capacity, efficiency and cyclability investigations.

\section{Experimental}

The NG powders were mixed with $10 \%$ PVdF binder in an NMP slurry, cast onto $25 \mu \mathrm{m} \mathrm{Cu}$ foil with loadings of 2-11 mg-active $/ \mathrm{cm}^{2}$ and dried under vacuum at $120^{\circ} \mathrm{C}$ for $12 \mathrm{~h}$. The electrodes were compressed by the bench top press between smooth plates at pressures of 100 , 200 or $300 \mathrm{~kg} / \mathrm{cm}^{2}$. Resistance measurements of the active mass layer were carried out with a novel four-point probe technique ${ }^{5}$. Electrochemical performance (IC and cyclability) was evaluated with $1.2 \mathrm{~cm}$ diameter electrodes assembled into metal Swagelok cells with Celgard separator, LP40 electrolyte from Merck (1M LiPF 6 in EC/DEC(1:1)), a Li RE and CE. Anodes were formed with two cycles at $\mathrm{C} / 25$ between $\mathrm{E}^{\mathrm{o}}$ or $1.0 \mathrm{~V}$ and $0.01 \mathrm{~V}$, with a taper charge at $0.01 \mathrm{~V}$. Cycling stability was checked with 100 cycles at $\mathrm{C} / 2$, over $70-80 \%$ of the $\mathrm{C} / 25$ full capacity. Limited capacity cycling will more accurately represent the cycling that would occur in a full cell. However, anode failure due to Li consumption in the SEI is not measurable with such a large excess of cycleable Li. 


\section{Results}

Anode Morphology Changes with Compression

The anodes studied are described in Table 1, along with manufacturer-supplied density and particle size information. Compression increased anode density by a factor of 1.6-2.5, depending on the graphite. As the amorphous fraction on the graphite increased the anode density achievable for a given pressure decreased. Anode porosities were calculated from the final thickness, decreasing from about $70 \%$ for the as-cast electrodes down to $30-50 \%$. It seems obvious that during compression in a standard roll-press, a certain amount of alignment will occur, especially when the particles have a high aspect ratio. However, with a bench-top press this might not be expected. In our companion work, there was a clear preferential compaction of the particles near the anode surfaces, i.e. the free surface exposed to the electrolyte and the surface next to the current collector [21]. This is predicted from theories on particle mechanics. The implications of a non-uniform morphology over the cross-section of the electrode on the conductivity measurements in currently being investigated.

\section{Conductivity Results}

Anode matrix resistivity and contact resistance were measured with a novel 4-point probe/calculation technique and reported elsewhere [21]. The resistivity results from this work are summarized in Figure 1 and Table 2. Also shown in the table are the data for the unpressed, low loading versions of these anodes, discussed previously [17]. The conclusions from the resistivity study were as follows. As expected, both addition of an amorphous carbon coating to the graphite and compression of the resulting composite significantly reduced the electrical resistivity of the resulting anode. The reduction in resistivity incurred by pressing decreased with an increase in amorphous coating content with the most significant reduction in resistivity occurring for the uncoated SL20 graphite with $\rho_{\text {unpressed }} / \rho_{300 \mathrm{~kg} / \mathrm{cm} 2}=7.9$. The contact resistance between the anode matrix and the current collector decreased with pressing for the uncoated graphite but increased with pressure for the coated materials. The best fit attained between the percolation model, developed to predict the resistivity of the anode from the properties of the components and an image analysis of the anode structure was achieved when it was assumed that the coatings on the graphite particles were somewhat permeable (i.e. soft). This particle-particle penetration results directly in an increase in the area of contact between particles.

The implications for anode conductivity are obvious when considering the construction of a high power Li-ion battery. However, if one considers that all of the matrix conductivities 
reported so far are on the order of 1000 times that of the ionic conductivity of the electrolyte, it is not always clear as to why we care. In reality, as stated above, this conductivity can change with cycling (not studied here) and it has been reported to affect the ICL/formation process in an anode. It is these connections we will examine in the remainder of this report.

\section{Anode Formation}

All of the anodes showed an OCV of around $3 \mathrm{~V}$ as assembled. Anodes were formed with the slow process as described above. Sample voltage profiles for the two C/25 cycles are shown in Fig. 2 for unpressed anodes of three types of graphite at a low and high loading. It is clear from these data that the amorphous carbon fraction on the GDR carbons led to a larger irreversible capacity in these electrodes. The reversible and total irreversible capacities were computed from these curves and summarized to show the effect of compression as shown in Fig. 3. The total irreversible capacity is taken as the sum of the ICL from the first two cycles and the reversible capacity is that from the second discharge (deintercalation of lithium). The first cycle portion is always the bulk of this value, or about $75 \%$ and the second cycle discharge capacity is usually a little higher than the first. The SL20 natural graphite shows a slight decrease in both reversible and irreversible capacity with increased anode density, as was reported in detail previously for thin electrodes [17]. For the GDR graphites, compression led to significant performance improvement with both increasing reversible capacity and decreasing ICL. The density ranges achievable with these carbons is also apparent from Figure 3. It appears that the performance of these carbons would benefit further even higher pressure calendaring.

In order to learn more about the formation process, the constant current formation data were differentiated to generate differential capacity $(\mathrm{dQ} / \mathrm{dV})$ curves. This type of analysis can reveal the thermodynamics of a process in a similar fashion to cyclic voltammetry (CV). However, unlike $\mathrm{CV}$, the capacitive effect in high surface area electrodes is absent since the applied current is constant. The first two formation cycles for the unpressed anodes of the three carbons are compared as $\mathrm{dQ} / \mathrm{dV}$ plots in two different potential ranges in Fig.4. The differential capacities are normalized to the weight of active material in the anode, correcting for the small differences in the loading $\left(6.8-8.6 \mathrm{mg} / \mathrm{cm}^{2}\right)$. The high-voltage region is blown up in the companion figures $4 \mathrm{~B}, 4 \mathrm{D}$, and 4F. No significant charge was passed until about $1.6 \mathrm{~V}$. The small high-voltage peaks at 1.6 and $1.25 \mathrm{vs} . \mathrm{Li} / \mathrm{Li}^{+}$are attributed to trace amounts of water and DEC, respectively. In all anodes, the first significant peak attributed to the formation of the SEI, or reduction of EC, was observed near $0.75 \mathrm{~V}$. 
The size and shapes of the solvent $\mathrm{dQ} / \mathrm{dV}$ peaks are clearly a function of the type of natural graphite. Some of the SL20 anodes showed two peaks near $0.75 \mathrm{~V}$. This could possibly be attributed to reduction on the edge and surface planes. The addition of amorphous carbon to the surface of graphite clearly reduces the size of these peaks. The $14 \%$ coated sample shows only a very small peak for the ICL formation. This suggests that the solvent is not able to reach the graphite surface, especially in the case of the $14 \%$ coated sample. This is the key reason these materials are of considerable interest for application with PC-containing electrolytes.

The onset of reversible Li intercalation begins at about the same potential as the solvent reduction peak, as can be seen in the right side plots in Fig. 4 by comparing the cathodic and anodic curves. The cathodic trace on the first formation cycle also shows that the irreversible electrochemical reaction is not limited to the solvent reduction peak but appears as well as an added background to the onset of the reversible process. The separation of the charge associated with these various processes will be discussed below.

Three major peaks are observed for the Li intercalation region below $0.3 \mathrm{vs}$. $\mathrm{Li} / \mathrm{Li}^{+}$. These are due to the well-known staging process [22], where the stage number refers to the number of empty graphene planes in between each layer filling with lithium. The difference between the three graphites lies primarily in the difference in peak potential and size for the first formation charge (cathodic portion), relative to the second cathodic process. The cathodic and anodic peak potentials for the second formation cycle are all separated by about $30 \mathrm{mV}$ and are similar for the three carbons. This magnitude of steady-state hysteresis is well-documented and attributed to the slow conversion between the solid-state stages [23]. The peak separation between the first-cycle anodic and cathodic processes show as much as double the value observed on the second cycle. This is more extreme in the coated carbons than in the SL20. The increased separation is due to the depression of the cathodic peaks, since the anodic peaks tend to line up quite well for the first two cycles. This is possibly due to the nature of the SEI formation. It is possible that this reaction is slower on the natural graphites, than on the more uniform particle morphology spherical graphites. The slowing of the surface reaction and/or an amorphous coating on the graphite will tend to extend the voltage range over which the surface reaction occurs and equilibrium will not be achieved by the time the bulk lithium intercalation begins. However, by the time the second cycle cathodic process begins, the surface layer formation is complete in a pseudo-equilibrium state. In the SL20 graphite, the electrochemical formation of this layer occurs more rapidly over a relatively narrow potential range, compared with the other carbons. The SEI formation on the coated natural graphites is further complicated by the fact that reaction an the amorphous fraction 
with the Li will not occur at the precise potentials observed with graphite. In fact disordered carbons show irreversible reaction occurs over the entire potential range being investigated here.

Different Contributors to the ICL

The first formation cycle for the three unpressed anodes are compared directly in Fig. 5. It is clear that the amorphous carbon coating tends to discourage the reaction of the graphite with the electrolyte components. This is the reason these carbons can function so well in PC-containing electrolytes. However, it is also clear that the total ICL for these carbons increases with the level of coating. Clearly the amorphous carbon fraction is reacting irreversibly with Li over a wide potential range. The capacity associated with the various processes was further quantified by determining the charge passed in various voltage windows. From Fig.s 4B, D and F, the capacity for the surface reaction is estimated as the difference in capacity between the first and second cathodic half-cycles between $1.0 \mathrm{~V}$ and $0.3 \mathrm{~V}$. This is similar to subtracting out the baseline process, or the portion of this capacity that is reversible. For the three unpressed anodes in Fig. 4, about 52,44 and $34 \%$ of the first-cycle ICL can be attributed to the high-potential region, for the SL20, GDR6 and GDR14 carbons, respectively. The fact that all of the ICL does not occur near the solvent reduction potentials has been discussed above. The amorphous fractions, either on the surface or within the graphite structure, will tend to isolate lithium deposits from deintercalation [24]. The higher fraction of the ICL occurring on the surface of the SL20 is expected since the quantity of the amorphous fractions in this carbon are expected to be lower than the coated graphites. This will be discussed further below.

\section{The Effect of Anode Compression}

Figure 6 shows first cycle differential capacity plots for the SL20 anode at the four levels of compression used in this study. No significant trends with anode density were observed in the potentials of the Li intercalation peaks for the first half-cycle. No significant trends were observed in the first cycle traces for the other carbons either. However, the random nature of these peaks does tend to subside a little with increased compression. Part of the issue with compression of these thick electrodes will be the non-uniform distribution of material on the cross-section of the anode. This will lead to a variable conductivity and subsequent non-uniform potential distribution over the cross-section. In addition, variable porosity could affect the accessibility of the solvent species for reaction with the graphite surfaces.

However, there is a much clearer trend on the high-potential regions for compression of the three carbons. While the SL20 anode shows little difference in this range with increased 
compression as shown in Figure 6b, compression clearly affects the coated graphites at high voltage, as shown in Figure 7. Compression of both the 6\% and 14\%-coated graphite anodes leads to increases in the electrolyte reduction on the graphite surface. This is most likely due to a disruption of the coating with compression of the anode. For the $14 \%$ coated carbon, the baseline reversible process in this potential window also increases somewhat with the level of compression (Fig. 7B). The disruption of the thick amorphous layer will lead to a lower resistance to Li transport to the graphite surface and higher reversible capacities.

The second cycle $\mathrm{dQ} / \mathrm{dV}$ curves for all three anode types at all compression levels are summarized in Fig. 8. The match-up in the potentials for the anodic and cathodic processes becomes much closer in the second cycle for the compressed anodes. A careful analysis of the peak positions with compression, reveals that while the absolute potentials of the individual peaks show insignificant trends with compression, the reversibility of a given stage improves, i.e. the potential difference between the cathodic and anodic peaks is reduced slightly, with an increase in anode density for the GDR carbons (see Fig. 9). This probably reflects the interpenetration of the amorphous coatings with increased compression and reduction in the diffusion pathway for lithium to migrate through this coating. This is opposite to the trend reported in previous work with thinner SL20 anodes, where we noted a decrease in the reversibility of the intercalation with increased compression, attributed to agglomeration of the graphite particles. This was clearly not observed with the thicker electrodes.

\section{Surface reaction dependence on density}

The fraction of the ICL corresponding to the surface reaction, calculated as above, is shown as a function of anode density for the three types of carbon anode in Fig. 10. This fraction decreases as the extent of coating increases, partly due to lower surface reaction (numerator) and partly due to the higher overall ICL (denominator) It is interesting that this fraction is independent of anode density for the SL20 anodes. This suggests that the amount of the area on the surface that reacts with the electrolyte is not changed by compression. It is also interesting to note that compression has a stronger influence on the surface reaction on the graphite, or inversely tends to decrease the charge consumed by the amorphous layers, possibly due to a lower surface area of amorphous carbon in contact with the electrolyte, or possibly due to the improved conductivity of the structure achieved through pressing. This is consistent with the model for the conductivity that showed a good fit, only when it was concluded that the surface coatings were overlapping with high levels of compression. This will improve with level of 
compression in a non-solvent intercalating electrolyte. However, this approach will be limited by the accessibility of the electrolyte to the graphite surface in a solvent that is known to destroy graphite, such as propylene carbonate.

\section{Cyclability}

Anode discharge capacity for the limited capacity $\mathrm{C} / 2$ cycling for the three types of anode at two levels of compression are shown in Fig. 11. In all cases, the cycle stability of the pressed anodes was greater than that for the unpressed anodes. The constant capacity portion of the data is due to the cycling protocol which limited the charge capacity to $85 \%$ of the theoretical capacity, to simulate the behavior in a real cell. The excess capacity (the remaining $15 \%$ ) is clearly consumed during cycling. After this point, the charge is limited by the tapering current at $10 \mathrm{mV}$ and the discharge capacity starts to drop with further degradation of the anode. The voltage profiles during an early cycle (\#2) and a cycle in the voltage-limited portion of the experiment are compared for an unpressed and a moderately $(200 \mathrm{~kg} / \mathrm{cm} 2)$ compressed SL20 anode in Fig. 12. The charge and discharge currents, along with the open-circuit periods where the voltage relaxes are also included in the figures. The voltage reached during the 15 minute open-circuit relaxation after the discharge is a useful measure of the relative impedance during discharge in these anodes. The final potential will be controlled by the residual lithium content in the anode. It is clear that more lithium was removed during the discharge of the pressed electrode (higher final voltage) than from the unpressed anode. After extensive cycling the impedance to lithium removal is increasing for both anodes, but the effect is worse for the unpressed electrode. Further analysis of these profiles would require a complex modeling effort and is beyond the scope of this work.

The voltage profiles for the compressed GDR anodes during $\mathrm{C} / 2$ cycling show similar trends with lower impedance during the early cycles and higher impedance for the later cycles. The cycling of the unpressed GDR anodes was poor from the very beginning. In addition, after testing these anode were observed to delaminate easily from the copper foil current collectors.

The stability of the capacity of a graphite anode during cycling will be controlled by many factors. We have shown that anode density affects the formation kinetics and charge consumed by the SEI layer, as well as the conductivity of the entire structure. These considerations will in turn affect the initial impedance of the structure. In addition, it is clear that initial anode density affects the rates of impedance rise in these anodes. It is possible that the same factors controlling the initial formation of the SEI are active during the continuous growth of the SEI during 
cycling, due to small electrolyte impurities. In this case, the improved SEI formation through improved anode conductivity will lead to lower rate SEI growth and thereby lower impedance rise during cycling. The more significant manifestation of these effects on the higher-loading (thicker) anodes could be simply due to the magnification of slightly non-uniform potential differences over the thicker electrodes.

\section{Conclusions}

Compression of both coated and uncoated natural graphite anodes tends to improve the reversible capacity, reduce the first cycle irreversibility loss on formation and vastly improve the cyclability of the anodes at moderate rates. The lower ICL for both coated and uncoated NG's is coincident with an improvement in the electronic conductivity of the anodes with compression. The extent ICL on the amorphous-carbon-coated graphites (GDR's) was higher than that on the un-coated SL20. The side-reaction resulting in this inefficiency occurs over a wide potential range, extending well below the $0.3 \mathrm{~V}$ onset of reversible lithium intercalation. The SL20 graphite also shows a significant amount of the irreversibility at low potentials, however more than $60 \%$ of the ICL can be attributed to the electrolyte reduction reaction. Anode compression showed minimal affects of the size (charge) or thermodynamics of the formation process on the SL20 graphite. However, compression of the coated graphite anodes lead to a more narrow potential range for SEI formation. However, this could be seen as a disadvantage in a PCcontaining electrolyte.

The compression of natural graphite anodes is critical their cycling stability. A moderately compressed SL20 anode showed excellent cycling stability out to 50 cycles in a lithium-metal half-cell, compared to the rapid impedance rises noted for the unpressed SL20 anode. The unpressed GDR-carbon anodes did not cycle at all well; compression greatly improved their performance, possibility related to potential distributions in the structure. Large changes in the impedance of the anodes to the discharge process with continued cycling were identified as the primary cause of the capacity fade. These impedance rises could be due to the continual formation of the SEI layer during cycling and respond to the improvements with compression in the same fashion as the formation process.

\section{Acknowledgements}

We acknowledge the receipt of the graphite materials from Superior Graphite and Mitsui Mining. Thanks for the helpful discussions of Yazami. This research was funded by the Assistant 
Secretary for Energy Efficiency and Renewable Energy, Office of FreedomCAR and Vehicle Technologies of the U.S. Department of Energy under Contract No. DE-AC03-76SF00098

\section{References}

1. M. Fujimoto, Y. Shoji, Y. Kida, R. Ohshita, T. Nohma, K. Nishio, J. Power Sources, 72 (1998) 226.

2. A. Naji, P. Willmann, D. Billaud, Carbon, 36 (1998) 1347.

3. J.O. Besenhard, M. Winter, J. Yang, and W. Biberacher, J. Power Sources, 54 (1995), 228.

4. D. Aurbach, B. Markovsky, I. Weissman, E. Levi, Y. Ein-Eli, Electrochim. Acta, 45 (1999) 67.

5. A.M. Andersson, M. Herstedt, A.G. Bishop, K. Edstrom, Electrochimica Acta, 47, (2002), 1885.

6. K. Zaghib, F. Brochu, A. Guerfi, K. Kinoshita, J. Power Sources, 103 (2001) 140.

7. E. Peled, D. Bar Tow, A. Merson, A. Gladkich, L. Burstein and D. Goldnitsky, J. Power Sources, 97-98, (2001) 52.

8. K. Zaghib, G. Nadeau, K. Kinoshita, J. Electrochem. Soc., 147 (2000) 2110.

9. R. Yazami, Electrochim. Acta 45, 87 (1999).

10. T. Takamura, M. Saito, A. Shimokawa, C. Nakahara, K. Sekine, M. Maeno, and N. Kibayashi, J. Power Sources, 90 (2000) 45. nec. For good SEI

11. G. Amatucci, A. DuPasquier, A. Blyr, T. Zheng and J.M. Tarascon, Electrochim. Acta 45 (1999) 255. Belcore, fast formation

12. M. Verbrugge and B. Koch, J. Electrochem. Soc. 146 (1999) 833. conductivity shouldn't be an issue

13. R. Brodd discussion

14. P. Novak, W. Scheifele, M. Winter and O. Haas, J. Power Sources 68 (1997) 267. compression

15. J. S. Gnanaraj, Y. S. Cohen, M. D. Levi, D. Aurbach, J. Electroanal. Chem. 516, 89 (2001).

16. J. Shim, K. Striebel, J. Power Sources 119-121 (2003) 934. Loading issue

17. J. Shim, and K. A. Striebel, J. Power Sources, accepted, Nov. 2003. Compression

18. C.-W. Wang, K.A. Cook and A. M. Sastry, , J. Electrochem. Soc. 150 (2003) A385. 
19. H. Wang, M. Yoshio, J. Power Sources, 93 (2001) 123 (the one with SEI decrease with coating increase)

20. H. Wang, T. Ikeda, K. Fukuda, M. Yoshio, J. Power Sources, 83 (1999) 141

21. C.-W. Wang, Y.-B. Yi, A. M. Sastry, J. Shim and K. A. Striebel, J. Electrochem. Soc., submitted, 2003.

22. T. Ohzuku, Y. Iwakoshi, K. Sawai, J. Electrochem. Soc., 140 (1993) 2490.

23. M. D. Levi and D. Aurbach, J. Electroanal. Chem. 421 (1997) 79.

24. T. Tsumura, A. Katanosaka, I. Souma, T. Ono, Y. Aihara, J. Kuratomi, M. Inagaki, Solid State Ionics, 135 (2000) 209. 
Figures.

1. Conductivity data from for three different natural graphite anodes as a function of coating density, $\triangle \longrightarrow$ GDR-14, $\times---$ GDR6, $\square$ - - - - SL20; (A) matrix resistivity, (B) contact resistance, [21].

2. Voltage profiles for the formation cycles $(\mathrm{C} / 25)$ of unpressed anodes with three different natural graphites:(A) thin anodes, about $5 \mathrm{mg} / \mathrm{cm}^{2}$ and (B) thick anodes, about 8.5 $\mathrm{mg} / \mathrm{cm}^{2}$.

3. Reversible (A) and irreversible (B) capacities measured for anodes from three different natural graphites as a function of anode density, $\triangle \longrightarrow$ GDR-14, $\times---$ GDR6, $\square-$ - - SL20.

4. Differential capacity plots of the two formation cycles for unpressed anodes from three different natural graphites. Data are normalized for differences in active material loading. (A,C,E) Lithium intercalation region, (B, D, F) hi voltage region: (A,B) SL20, (C,D) GDR6 and (E,F) GDR14.

5. Differential capacity plots of the hi voltage region for the first formation cycle for unpressed anodes from three different natural graphites. Data are normalized for differences in active material loading.

6. First cycle differential capacity plots of the SL20 natural graphite anodes at different levels of compression :(A) Li intercalation region, (B).Expanded scale hi voltage region.

7. Expanded scale hi voltage portion of the first cycle differential capacity plots of the amorphous-coated natural graphites anodes at different levels of compression :(A) GDR6, (B) GDR14, darker lines correspond to higher compression levels.

8. Li intercalation portion of the second cycle differential capacity plots of three natural graphites anodes at different levels of compression :(A) SL20, (B) GDR6, (C) GDR14. 
Darkness of line corresponds to higher compression.

9. Li intercalation peak reversibility as a function of anode compression for three lithium intercalation stages: (A) SL20, (B) GDR6, (C) GDR-14.

10. Fraction of the ICL accounted for by the surface reaction from the formation data as a function of anode density; $\triangle \longrightarrow$ GDR-14, $\diamond---$ GDR6, $\square$ - - - - SL20; .

11. Discharge capacity for pressed and unpressed anodes prepared from three different natural graphites. Charge capacity was limited to $85 \%$ of formation capacity; SL20: $\diamond$, $\diamond$; GDR6: $\triangle, \mathbf{\Delta}$; GDR14: $\bigcirc, \boldsymbol{\bullet}$, open symbols=unpressed, closed symbols=pressed.

12. Voltage profiles during cycle \#2 ( $\square$ ) and a cycle in the voltage-limited portion of the experiment $(\diamond)$ are compared a moderately compressed $\left(200 \mathrm{~kg} / \mathrm{cm}^{2}\right)$ SL20 anode. 
Table 1 Physical Properties of natural graphites

\begin{tabular}{|c|c|c|c|}
\hline Anode & D50 $\mu \mathbf{m}$ & SA (m²/g) & Morphology \\
\hline GDR6 & 30.2 & 2.4 & Round, 6\% coated \\
\hline GDR14 & 26.2 & 1.5 & Round, 14\% coated \\
\hline SL20 & 22 & $<4.6$ & Potatoes \\
\hline
\end{tabular}

Table 2 Properties of the Natural Graphite Anodes

\begin{tabular}{|c|c|c|c|c|c|}
\hline Anode & $\begin{array}{l}\text { Loading } \\
\left(\mathrm{mg} / \mathrm{cm}^{2}\right)\end{array}$ & $\begin{array}{l}\text { Density } \\
\left(\mathrm{g} / \mathrm{cm}^{3}\right)\end{array}$ & $\begin{array}{c}\text { Thickness } \\
(\mu \mathrm{m})\end{array}$ & $\begin{array}{c}\text { Matrix Res*. } \\
(\Omega-c m)\end{array}$ & $\begin{array}{c}\text { Contact. Res*. } \\
\left(\mathrm{m} \Omega-\mathrm{cm}^{2}\right)\end{array}$ \\
\hline SL20-04 & 5.5 & $0.7-1.3$ & $80-100$ & 0.77 & $4.0 \times 10^{6}$ \\
\hline SL20-02 & 16.0 & $0.9-2.1$ & $65-160$ & 0.51 & $3.9 \times 10^{6}$ \\
\hline GRD6-01 & 4.2 & 0.6 & 70 & 0.11 & $1.7 \times 10^{4}$ \\
\hline GDR6-02 & 8.6 & $0.7-1.7$ & $75-125$ & 0.15 & $3.3 \times 10^{4}$ \\
\hline GDR14-01 & 4.8 & 0.6 & 80 & 0.014 & $8.2 \times 10^{2}$ \\
\hline GDR14-02 & 8.5 & $0.7-1.4$ & $80-130$ & 0.039 & $4.2 \times 10^{3}$ \\
\hline
\end{tabular}

* for the unpressed anodes 

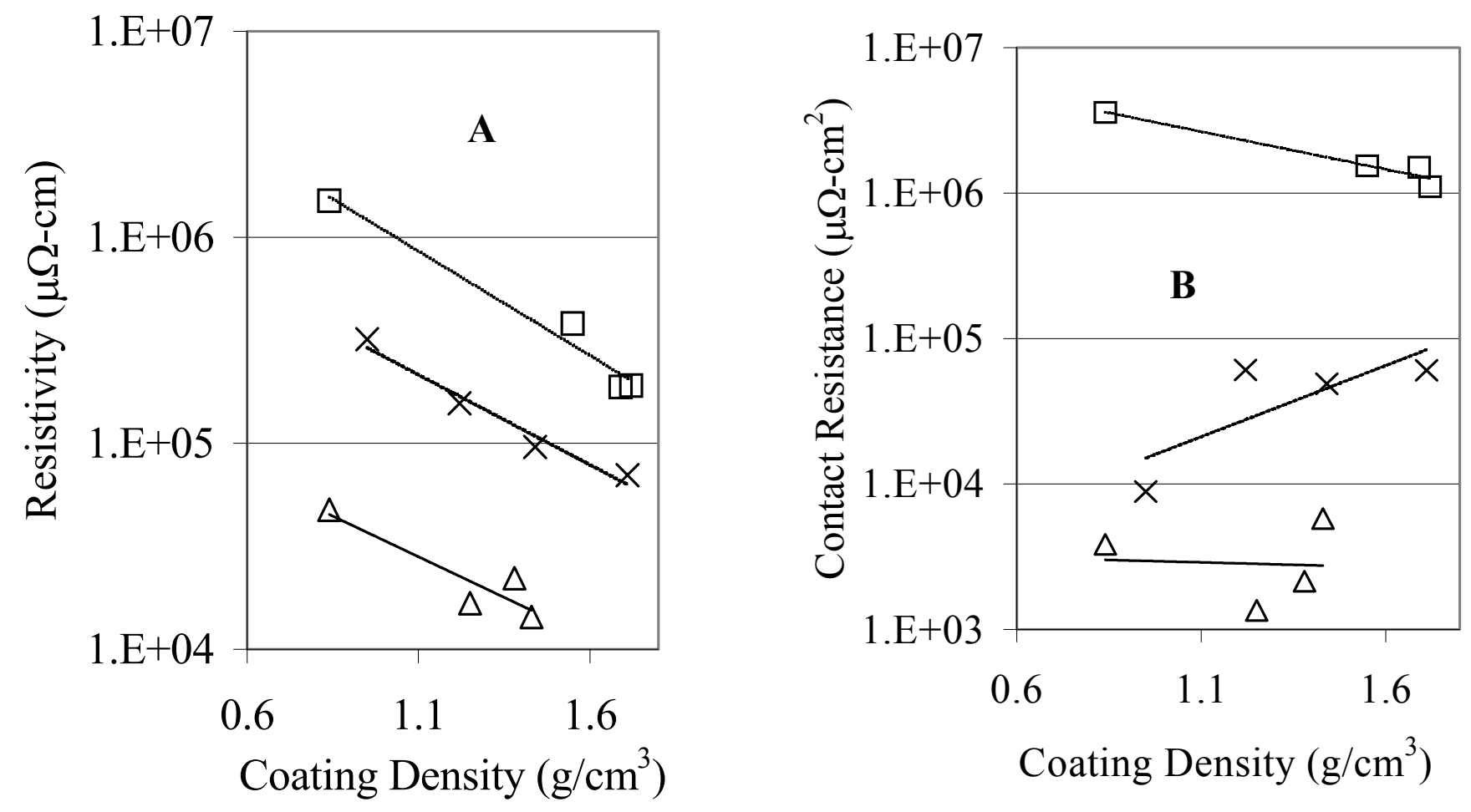

Figure 1 

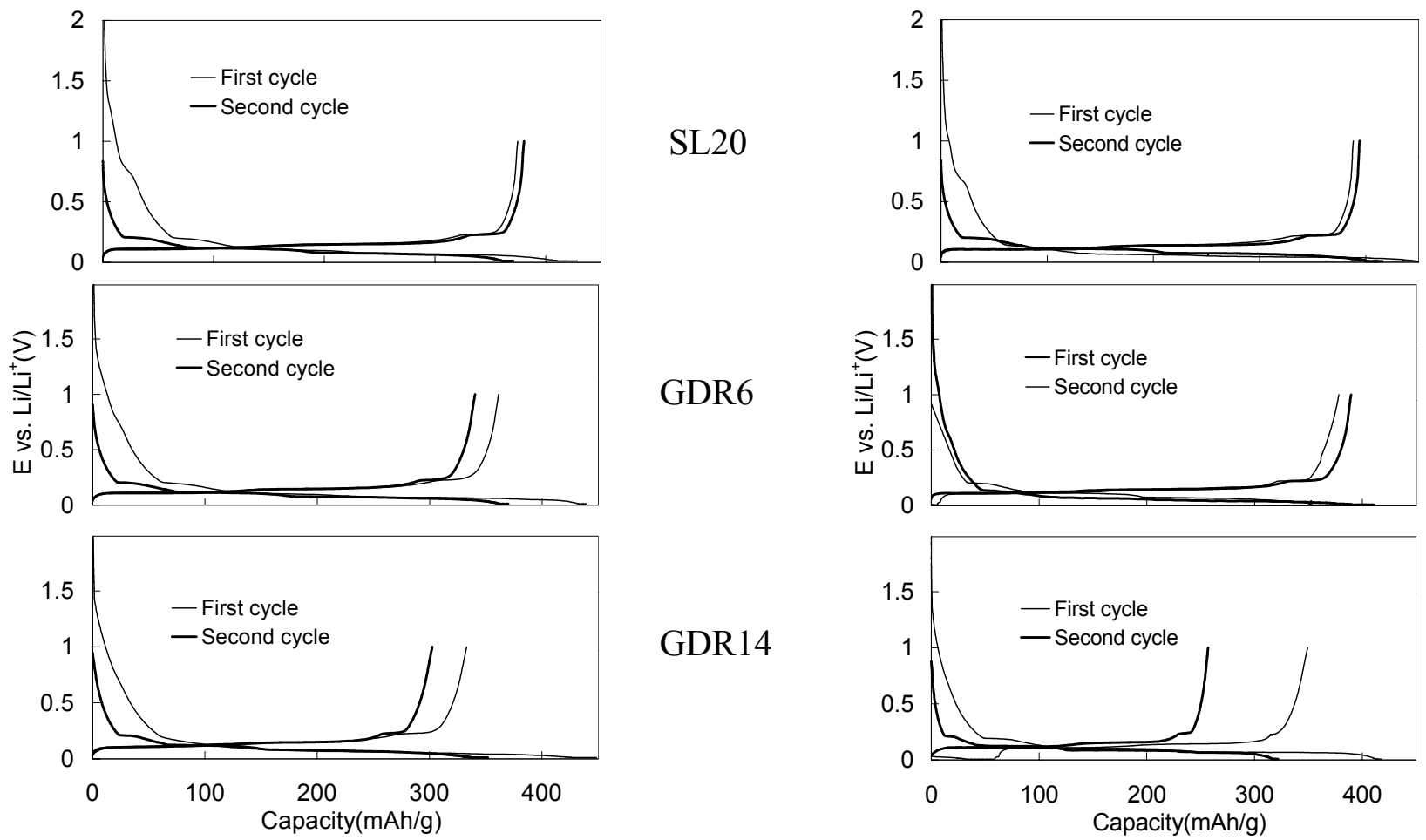

A

Figure 2 